International Journal of Pure and Applied Mathematics

Volume 97 No. $4 \quad 2014,543-549$

ISSN: 1311-8080 (printed version); ISSN: 1314-3395 (on-line version)

url: http://www.ijpam.eu

doi: http://dx.doi.org/10.12732/ijpam.v97i4.13

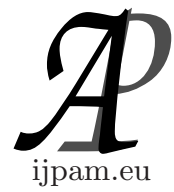

\title{
RELATIVE HEAT LOSS REDUCTION FORMULA FOR WINDOWS WITH MULTIPLE PANES
}

\author{
Cassandra Reed ${ }^{1}$, Jean Michelet Jean-Michel ${ }^{2} \S$ \\ ${ }^{1,2}$ Department of Mathematics and Computer Science \\ South Carolina State University \\ Orangeburg, SC 29117, USA
}

\begin{abstract}
We derive an expression for the heat flux through a window constructed with multiple panes of glass separated pairwise by air layers of a given thickness. We compute the relative heat loss reduction achieved in comparison to a window with no air gap and the same amount of glass. We examine how the relative heat loss reduction function behaves when we scale the number of panes up to a large value.
\end{abstract}

AMS Subject Classification: 65K10, 78M50, 80A20

Key Words: heat flux reduction, heat transfer, heat flux equilibrium

\section{Introduction}

In this model, warm air escapes from a heated building through a window. The window comprises $n$ glass panes of thickness $d$ separated pairwise by a layer of air of width $L$.

We assume that the inside temperature $T_{1}$ is greater than the outside temperature $T_{2}$, reflecting the fact that heat flows from the inside to the outside. In the case $n=2$, we follow Mesterton-Gibbons [1]. If we indicate by $x=0$ the interface of the inside air and the inside window pane, then the outside pane and outside air meet at $x=2 d+L$. Let $T(x)$ be the temperature at $x$. Thus we have $T(0)=T_{1}$ and $T(2 d+L)=T_{2}$. We also write $T(d)=T_{A}$ and $T(d+L)=T_{B}$ for the other two air-glass interfaces in between.

Received: August 30, 2014

(c) 2014 Academic Publications, Ltd.

$\S$ Correspondence author url: www.acadpubl.eu 
The heat flows along $x$ according to Fick's law

$$
F(x)=-k(x) \frac{d T}{d x}
$$

where $F(x)$ stands for the heat flux per unit area across the window at point $x$ and the function $k(x)$ represents the thermal conductivity of the medium at $x$. Fick's law simply states that heat flux at a point $x$ is proportional to the temperature gradient at $x$; The steeper the gradient, the higher the heat flow.

In the two-pane model, for example, $k$ is a piecewise constant function that takes the value $K_{A}$ for $x$ between $d$ and $d+L$, the points $x$ that lie in the air gap. For $x$ in the remaining intervals $0<x<d$, $d+L<x<2 d+L$, in the glass medium, we assign to $k$ the value $k_{G}$.

In this paper, we are interested in determining the heat flux across the glass and air for an n-glazed window once the flow has reached a constant equilibrium. In Section 2, some straight-forward computations as found in [1] yield the equilibrium value the flux $F$ settles to in the case $n=2$. We use these computations in Section 3 to treat the case $n=3$. Formulas for the flux and relative heat flux reduction for a window with $n$ panes follow in Section 4 . We conclude our investigation with Section 5.

\section{Double-Glazed Window}

As in [1], integrating with respect to $x$ the expression for Fick's law over the interval $0<x<d$, we obtain

$$
F(x)=K_{G} \frac{T_{1}-T_{A}}{d} .
$$

Repeating the process over the remaining intervals and equating the expressions for the respective heat fluxes so obtained, we have the chain of inequalities

$$
K_{G} \frac{T_{1}-T_{A}}{d}=K_{A} \frac{T_{A}-T_{B}}{L}=K_{G} \frac{T_{B}-T_{2}}{d}=F .
$$

Solving for $T_{A}$ and $T_{B}$ in terms of $T_{1}$ and $T_{2}$ gives

$$
\begin{aligned}
T_{A} & =\frac{\left(\frac{K_{G} L}{K_{A} d}+1\right) T_{1}+T_{2}}{\frac{K_{G} L}{K_{A} d}+2} \\
T_{B} & =\frac{T_{1}+\left(\frac{K_{G} L}{K_{A} d}+1\right) T_{2}}{\frac{K_{G} L}{K_{A} d}+2} .
\end{aligned}
$$


Therefore, the flux across across the double-paned window eventuallys settles down to an equilibrium value

$$
F=F_{2}=\frac{K_{G}}{d} \frac{T_{1}-T_{2}}{2+\frac{K_{G} L}{K_{A} d}}
$$

which, in the $L=0$ limit, becomes

$$
F_{2 s}=\frac{K_{G}}{d} \frac{T_{1}-T_{2}}{2}
$$

where $F_{2 s}$ is the rate of heat loss for a single-paned window with the same amount of glass.

Thus, by setting the glass panes a distance of $L$ units apart, we achieve a relative reduction of heat loss

$$
\Delta_{2}=\frac{F_{2 S}-F_{2}}{F_{2 S}}=\frac{\frac{K_{G} L}{K_{A} d}}{2+\frac{K_{G} L}{K_{A} d}} .
$$

The thermal conductivity of glass at room temperature varies between $4 \times$ $10^{-3}$ and $8 \times 10^{-3} \mathrm{~J} / \mathrm{cm} . \mathrm{sec} .{ }^{\circ} \mathrm{C}[2]$, while the thermal conductivity of dry air is approximately $2.5 \times 10^{-4} \mathrm{~J} / \mathrm{cm} . \mathbf{s e c} .{ }^{\circ} \mathrm{C}[3]$. Therefore, the ratio $\frac{K_{G}}{K_{A}}$ varies between 16 and 32 , which means that a window with a conductivity ratio of 16 and an air gap of four pane-widths already achieves a heat loss reduction of $97 \%$. The heat reduction function $\Delta_{2}$ is a strictly increasing function of the gap aspect ratio $L / d$, and approaches rapidly the limiting value 1 .

Manufacturers know this, which is why we do not often see windows with 4 or 5 glass panes on the market. Nevertheless, how the heat loss reduction function scale for a window with a large number, $n$, of glass panes remains an interesting theoretical question. How does $\Delta$ depend on $n$ and the gap aspect ratio $L / d$ ?

Specifically, we address the following situation. Instead of two, we take $n$ glass panes of thickness $d$ each and put an air gap of thickness $L$ between each successive pair to form an $n$-glazed window. Compared to a single-paned window of thickness $n d$, how much heat loss reduction is achieved? What form does the function $\Delta_{n}$ take?

We take an inductive approach to this question. Determining $\Delta_{3}$ will allow us to infer the form of $\Delta_{n}$ for a general $n$. 


\section{Triple-Glazed Window}

With a triple-glazed window, we have air-glass interfaces at $x=0, d, d+L, 2 d+$ $L, 2 d+2 L, 3 d+2 L$ that are kept at temperatures $T_{1}, T_{A}, T_{B}, T_{C}, T_{D}, T_{3}$, respectively. Integrating Fick's law as we did in the last section leads us this time to the string of equations

$$
\begin{aligned}
K_{G} \frac{T_{1}-T_{A}}{d} & =K_{A} \frac{T_{A}-T_{B}}{L}=K_{G} \frac{T_{B}-T_{c}}{d}= \\
K_{A} \frac{T_{C}-T_{D}}{L} & =K_{G} \frac{T_{D}-T_{3}}{d}=F_{3},
\end{aligned}
$$

where $F_{3}$ denotes the heat flux through the three-pane window.

Part of Equations (1) can be written as

$$
K_{G} \frac{T_{3}-T_{D}}{d}=K_{A} \frac{T_{D}-T_{C}}{L}=K_{G} \frac{T_{C}-T_{B}}{d}=-F_{3},
$$

which we can, as we did in the previous section, solve for $T_{D}$ and $T_{C}$ to find

$$
\begin{aligned}
& T_{D}=\frac{(\rho+1) T_{3}+T_{B}}{\rho+2} \\
& T_{C}=\frac{T_{3}+(\rho+1) T_{B}}{\rho+2},
\end{aligned}
$$

if we set $\rho=\frac{K_{G} L}{K_{A} d}$.

On the other hand, in (1) we also have

$$
K_{G} \frac{T_{1}-T_{A}}{d}=K_{A} \frac{T_{A}-T_{B}}{L}=K_{G} \frac{T_{B}-T_{c}}{d}=F_{3},
$$

from which we deduce

$$
\begin{aligned}
& T_{A}=\frac{(\rho+1) T_{1}+T_{C}}{\rho+2}, \\
& T_{B}=\frac{T_{1}+(\rho+1) T_{C}}{\rho+2} .
\end{aligned}
$$

Next, we use the expressions for $T_{B}$ and $T_{C}$ to obtain

$$
(\rho+2) T_{B}=T_{1}+(\rho+1) T_{C}=T_{1}+\frac{\rho+1}{\rho+2} T_{3}+\frac{(\rho+1)^{2}}{\rho+2} T_{B},
$$


which yields

$$
T_{B}=\frac{\rho+2}{2 \rho+3} T_{1}+\frac{\rho+1}{2 \rho+3} T_{3} .
$$

This allows us to find

$$
T_{D}=\frac{T_{1}+2(\rho+1) T_{3}}{2 \rho+3} .
$$

Finally, inserting $T_{D}$ into the last of the equations in (1), we arrive at the formula for the heat flux through a three-pane window:

$$
F_{3}=\frac{K_{G}}{d}\left[\frac{T_{1}+2 T_{3}\left(1+\frac{K_{G} L}{K_{A} d}\right)}{3+2 \frac{K_{G} L}{K_{A} d}}-T_{3} .\right.
$$

It is, as one would expect, a function of the the inside and outside air temperatures. This time again, the temperatures at the inner air-glass interfaces do not influence the flow at equilibrium.

\section{Window with n Panes}

From the expression obtained in the last section for the heat flux for a window with 3 panes, we infer the flux for an $n$-glazed window to be of the form

$$
F_{n}=\frac{K_{G}}{d}\left[\frac{T_{1}+(n-1) T_{n}\left(1+\frac{K_{G} L}{K_{A} d}\right)}{n+(n-1) \frac{K_{G} L}{K_{A} d}}-T_{n}\right],
$$

where $T_{1}$ and $T_{n}$ are the inside and outside air temperatures, respectively.

In the $L=0$ limit, $F_{n}$ reduces to

$$
F_{n s}=\frac{K_{G}}{d} \frac{T_{1}-T_{n}}{n},
$$

with $F_{n s}$ denoting the flux across a single-paned window with the same amount of glass.

The relative heat loss reduction is given by

$$
\Delta_{n}=\frac{F_{n S}-F_{n}}{F_{n S}}=\frac{(n-1) \frac{K_{G} L}{K_{A} d}}{n+(n-1) \frac{K_{G} L}{K_{A} d}} .
$$


As a sequence of functions of the gap aspect ratio $\frac{L}{d}, \Delta_{n}$ converges to the limit $\frac{\frac{K_{G} L}{K_{A}}}{1+\frac{K_{G} L}{K_{A}}}$ as $n$ gets large. As for the behavior of that sequence, that limit is reached in a monotonic fashion, since we have

$$
\frac{d}{d n} \Delta_{n}=\frac{\frac{K_{G} L}{K_{A} d}}{\left[n+(n-1) \frac{K_{G} L}{K_{A} d}\right]^{2}},
$$

which remains strictly positive for all positive values of the gap aspect ratio $\frac{L}{d}$. Therefore, the absolute ceiling on the amount of relative heat loss reduction achievable is $\frac{\frac{K_{G} L}{K_{A} d}}{1+\frac{K_{G} L}{K_{A}}}$, no matter the number of panes used. The monotonic convergence of the sequence $\Delta_{n}$ conforms to one's intuition that the more panes and the wider the gaps, the more energy is saved.

\section{Conclusion}

In an inductive approach, using the expression for the heat flux through a double-glazed, then the heat flux through a triple-glazed window, we inferred the expression for the heat flux through a window with $n$ panes of thickness $d$ set a distance of $L$ units apart. We obtained a formula for the relative heat loss reduction $\Delta_{n}$ as the relative difference between the heat flux through an $n$-glazed window and that of a single-paned window with the same amount of glass. As a function of the gap aspect ratio $\frac{L}{d}$, we found that the amount of relative heat loss reduction $\Delta_{n}$ achieved starts at $\frac{\frac{K_{G} L}{K_{A}}}{2+\frac{K_{G} L}{K_{A} d}}$ when $n$ equals 2 , increases monotonically with $n$, and approaches the limiting value $\frac{\frac{K_{G} L}{K_{A} d}}{1+\frac{K_{G} L}{K_{A} d}}$.

\section{References}

[1] M. Mesterton-Gibbons, A Concrete Approach to Mathematical Modelling, Wiley, New York (1995).

[2] L.D. Landau, A.I. Akhiezer, E.M. Lifshitz, General Physics: Mechanics and Molecular Physics, Pergamon Press, New York (1967). 
[3] G.K. Batchelor, An Introduction to Fluid Dynamics, Cambridge University Press, Cambridge (1967). 
